\title{
The Application of Different Breathing Training Methods in Elderly Patients With Femoral Neck Fracture: A Prospective, Randomized, and Single- Blind Study
}

\section{Yan Zhang}

Affiliated Zhongshan Hospital of Dalian University

Hong Wang (D1752322696@qq.com )

Affiliated Zhongshan Hospital of Dalian University https://orcid.org/0000-0003-1153-0384

Mei Jing Fan

Affiliated Zhongshan Hospital of Dalian University

Lin Fan

Affiliated Zhongshan Hospital of Dalian University

Min Wang

Affiliated Zhongshan Hospital of Dalian University

\section{Research article}

Keywords: Old age, Femoral neck fracture, Lung function training, Lung function trainers, Forced expiratory volume in the first second, Forced vital capacity, Blood oxygen saturation, Pulmonary complications, Hospitalization days

Posted Date: October 13th, 2021

DOI: https://doi.org/10.21203/rs.3.rs-958980/v1

License: (c) (i) This work is licensed under a Creative Commons Attribution 4.0 International License. Read Full License 


\section{Abstract}

Purpose: Femoral neck fractures in elderly patients lead to reduced mobility and increased risk of pulmonary complications. The main purpose of this study is to observe the forced expiratory volume in the first second(FEV1), forced vital capacity $(\mathrm{FVC})$, blood oxygen saturation $\left(\mathrm{SPO}_{2}\right)$, pulmonary complications, and hospitalization days in elderly patients with femoral neck fractures, and to evaluate the use of lung function trainers to train elderly patients' lung function effect and feasibility.

Methods: This is a prospective, randomized and single-blind study. 108 elderly patients with femoral neck fractures over 70 years old were randomly divided into a conventional group and a lung function trainer group. The conventional group received effective cough training and lip-reducing breathing training, and the lung function trainer group received effective cough training and lung function training. Until 2 weeks after surgery. The main outcomes are forced expiratory volume in the first second, forced vital capacity, and blood oxygen saturation. The secondary outcomes are pulmonary complications and the hospitalization days.

Results: 50 subjects in the conventional group and 51 subjects in the lung function trainer group completed the study. On the 3rd and 7th day of training, the FVC of the lung function trainer group was higher than that of the conventional group $(2.94 \pm 0.40 v s .2 .72 \pm 0.34, P<0.05 \rrbracket$

$3.02 \pm 0.40 v s .2 .86 \pm 0.34, P<0.05)$, and the FVE1 of the the lung function trainer group was higher than that of the conventional group (1.99 $\pm 0.37 \mathrm{vs} .1 .80 \pm 0.42 \varangle \mathrm{P} \otimes 0.05 \llbracket 2.14 \pm 0.39 \mathrm{vs} .1 .94 \pm 0.43, \varangle 0.05)$, and the blood oxygen saturation of the lung function trainer group was higher than that of the conventional group ( $97.10 \pm 1.35 \mathrm{vs} .96 .28 \pm 1.21 \otimes 97.47 \pm 1.35 \mathrm{vs} .96 .86 \pm 1.36, P \llbracket 0.05), 4$ cases of pneumonia in the conventional group, no one in the lung function trainer group was diagnosed with pneumonia, and the hospitalization days in the lung function trainer group was less than that in the conventional group $(15.55 \pm 1.45$ VS.17.2 $\pm 2.58, P<0.05)$.

Conclusion: For elderly patients with femoral neck fracture, the use of lung function training device to strengthen respiratory muscle function training during perioperative period can improve the patient's lung function in a short period of time, reduce the complications of respiratory system infection, and shorten the hospitalization days. It is worthy of clinical application.

\section{Introduction}

Femoral neck fractures are a common clinical trauma and often occur in elderly patients. With the increase of life expectancy, the incidence rate is also increasing, especially in developed countries and regions where the elderly population is increasing rapidly. According to Melton's statistics ${ }^{[1]}$, the number of hip fractures worldwide was 10,000 in 1990. By 2025, this number is expected to reach 4 million and by 2050 it can reach 6.3 million. In the United States, there are currently about 329,000 hip fractures in the elderly each year, and the number will exceed 500,000 by $2040^{[2]}$. Due to the progressive decline of vital life organs to varying degrees, coupled with anesthesia, surgical trauma, pain and other reasons, elderly 
patients can easily cause perioperative respiratory failure, and have a higher probability of pulmonary complications. Respiratory failure is ultimately life-threatening. Although conventional respiratory function training (Knocking back, Coughing, and Expectoration) can improve the lung function of patients after

surgery to varying degrees ${ }^{[3]}$, but the effect on elderly patients is not ideal ${ }^{[4]}$. How to reduce the incidence of pulmonary complications in elderly patients during perioperative period, early prevention and postoperative strengthening of lung care are particularly important ${ }^{[5]}$. Respiratory function training is a new technique applied to patients undergoing perioperative surgery of the chest and abdomen in recent years. After breathing function training, it can enhance the patient's tolerance to surgery, improve the lung function of postoperative patients, and reduce postoperative pulmonary complications. Incidence ${ }^{[6-7]}$. This study compared the effects of different breathing training methods on perioperative prevention of pulmonary complications in elderly patients with femoral neck fractures, and provided an effective and reasonable training method for clinical care. The report is as follows.

\section{Materials And Methods}

\section{Design overview}

This is a prospective, randomized, single-blind clinical study, approved by the Ethics Committee of Zhongshan Hospital Affiliated to Dalian University.

\section{Subjects}

This study recruited 108 elderly patients over 70 years of age who had unilateral femoral neck fractures and planned to undergo artificial total hip replacement surgery. The inclusion criteria as follows: (1) The preoperative lung function test results should meet one of the following criteria as a minimum: forced expiratory volume in the first second (FEV1) $>2 \mathrm{~L}$, forced expiratory volume in the first second/forced vital capacity (FEV1/FVC) $>50 \%$; Maximum minute ventilation (MVV) $>80 \mathrm{~L} / \mathrm{min}$ or $>50 \%$ of predicted value; Residual air volume/Maximum minute ventilation (RV/TIC) $<50 \%$, estimated postoperative FEV1 $>0.8 \mathrm{~L} ;(2)$ First admission Arterial blood gas $\mathrm{PaO} 2$ in resting state: $60-70 \mathrm{mmHg}$; (3) No lung infection. The exclusion criteria were as follows: (1) Patients with severe comorbidities such as heart, brain, liver, and kidney before surgery; (2) Patients with severe mental illness; (3) Arterial blood gas PaO2>71 $\mathrm{mmHg}$ in the first resting state of admission. All patients were randomly divided into a control group ( 54 cases) and a lung function training device group (54 cases). In addition, patients with unexpected or critical situations during treatment withdrew from the study.

\section{Program}

All measured values are measured by the same nurse (blind) using a portable spirometer to measure all patients (blind) FEV1, FVC, MVV, RV/TIC, PaO2,SPO2, the incidence of intrapulmonary infection within two weeks, and the hospitalization days. All interventions are carried out by professionally trained nurses (not blind). 


\section{Conventional group(CG)}

Receive lip constriction breathing training and effective cough training.Lip constriction breathing training: The technique of reduced lip breathing is to extend the expiration time, increase the airway pressure, and delay the collapse of the airway through the weak resistance formed by the shrinking lip. The patient closes the mouth and breathes in through the nose, then slowly exhales through the shrinking lip (whistlelike) while contracting the abdomen. The ratio of exhalation to inhalation time is $1: 2$ or $1: 3$. The size of the shrinking lip and the expiratory flow rate are suitable to be able to make the candle flame that is 15$20 \mathrm{~cm}$ away from the lip and level with the height of the lip incline with the airflow without being extinguished. Effective cough training: The patient takes the supine position, the upper body can be slightly forward, inhale slowly and deeply, hold the breath for a few seconds, and then cough 2-3 times, contract the abdominal muscles when coughing, retract the abdominal wall, or press with their own hands On the upper abdomen, help cough, stop coughing, shrink the lips to exhale as much as possible, inhale slowly and deeply, repeat the above actions, do it 2-3 times, rest for a few minutes and start again.

\section{Lung function trainer group(LG)}

Receive effective cough training and use lung function trainer for respiratory muscle function training. The professionally trained nurses introduces the lung function training device to the patient, informs the patient of the precautions and teaches the patient to use it. How to use:(1)Take out the lung function trainer, connect the threaded connection tube with the interface and bite of the shell, and place it vertically; (2)Inspiratory training: the base with the word "suction" is placed below, breathing in the mouth, breathing in with a deep and even flow rate of inspiration to keep the floating ball rising, and as long as possible to keep it, after 10-15 minutes of inspiratory training, to rest with normal breathing;(3)Exhalation training: place the base with the word "blow" on the bottom, do exhalation training, with deep and even exhalation flow rate to keep the floating ball rising, and keep it as long as possible, after 10-15 minutes of exhalation training, rest with normal breathing .

\section{Results}

Fifty-one subjects (age, $73.87 \pm 4.18$ years old) in the $L G$ and 50 subjects (age, $74.7 \pm 4.061$ years old) in the CG completed the study, of which 7 patients were discharged prematurely and withdrawal from the trial due to electrolyte imbalance, postoperative delirium, or arrhythmia. The general information used to treat subjects is shown in table 1.

Table 1 Comparison of general information in the two groups of patients. 


\begin{tabular}{|lllll|}
\hline & $\begin{array}{l}\text { Control } \\
\mathrm{n}=50\end{array}$ & $\begin{array}{l}\text { Experiment } \\
\mathrm{n}=51\end{array}$ & $\mathrm{t} \rrbracket \mathrm{\chi} 2$ & $\mathrm{P}$ \\
\hline Age (years) & $74.66 \pm 3.68$ & $74.63 \pm 3.47$ & 0.05 & 0.96 \\
\hline Sex, female $(\mathrm{n}, \%)$ & $26 \rrbracket 52 \rrbracket$ & $29 \llbracket 56.86 \rrbracket$ & 0.24 & 0.62 \\
\hline Complications & & & & \\
\hline diabetes & 6 & 5 & 0.44 & 0.51 \\
\hline hypertension & 20 & 18 & 0.48 & 0.48 \\
\hline Pa02 & $65.14 \pm 2.81$ & $64.84 \pm 3.01$ & 1.51 & 0.61 \\
\hline
\end{tabular}

\section{Spirometer tests}

The FVC at three time points is shown in Table 2.The results of repeated measurement ANOVA showed that the difference of $F V C$ at different time was statistically significant $(F=42.43, P<0.001)$. FVC increased gradually with the extension of time. There was no significant difference in FVC between the two groups of patients at admission $(t=1.05, P=0.30)$. The $F V C$ of the $L G$ was higher than that of the $C G$ at 3 days after operation ( $t=-3.07, P<0.05), 7$ days after operation the $F V C$ of the $L G$ is higher than that of the CG(t=-2.16,P<0.05)

Table 2 FVC comparison

\begin{tabular}{|lllllll|}
\hline & & The day of admission & After 3 days & After 7 days & $F$ & P value* \\
Group & $n$ & & & & & \\
\hline CG & 50 & $3.1 \pm 0.36$ & $2.72 \pm 0.34$ & $2.86 \pm 0.34$ & 42.43 & $<0.001$ \\
\hline LG & 51 & $3.02 \pm 0.40$ & $2.94 \pm 0.40$ & $3.02 \pm 0.40$ & & \\
\hline
\end{tabular}

*P-value for repeated ANOVA.

The FEV1 at three time points is shown in Table 3. The results of repeated measurement ANOVA showed that the difference of FEV1 at different time was statistically significant $(F=21.26, P<0.001)$. FEV1 increased gradually with the extension of time. There was no statistically significant difference in FVE1 between the two groups at admission $(t=0.24 \llbracket P=0.81)$. $F V E 1$ in the $L G$ was higher than that in the $C G$ at 3 days after operation $(t=-2.324, P<0.05), 7$ days after operation the $F V E 1$ of the $L G$ was higher than that of the CG $(\mathrm{t}=-2.405 \bigotimes \mathrm{P}<0.05)$

Table 3 Comparison of FEV1 


\begin{tabular}{|c|c|c|c|c|}
\hline Group & $\mathrm{n}$ & The day of admission & After 3 days & After 7 days \\
\hline CG & 50 & $2.18 \pm 0.46$ & $1.80 \pm 0.42$ & $1.94 \pm 0.43$ \\
\hline LG & 51 & $2.16 \pm 0.39$ & $1.99 \pm 0.37$ & $2.14 \pm 0.39$ \\
\hline
\end{tabular}

*P-value for repeated ANOVA.

\section{Blood oxygen saturation(SPO2)}

The blood oxygen saturations at the three time points are shown in Table 4. The results of repeated measurement ANOVA showed that the difference of SPO2 at different time was statistically significant ( $F$ $=8.433, \mathrm{P}<0.001)$. SPO2 increased gradually with the extension of time. There was no statistically significant difference in SPO2 between the two groups before surgery $(t=0.755 \otimes P=0.45)$, and the SPO2 in the LG was higher than that in the CG $(t=-3.207, P<0.05)$ after 3 days. 7 days after operation the SPO2 in the $L G$ was higher than that in the $C G(t=-2.271 \otimes P<0.05)$

Table 4 Comparison of blood oxygen saturation (\%)

\begin{tabular}{|c|c|c|c|c|}
\hline Grol & $\mathrm{n}$ & The day of admission & After 3 days & After 7 days \\
\hline CG & 50 & $98.14 \pm 1.56$ & $96.28 \pm 1.21$ & $96.86 \pm 1.36$ \\
\hline LG & 51 & $97.9 \pm 1.63$ & $97.10 \pm 1.35$ & $97.47 \pm 1.35$ \\
\hline
\end{tabular}

*P-value for repeated ANOVA.

\section{Pneumonia, hospitalization days}

The comparison of pneumonia and hospitalization days between the two groups is shown in Table 5 . After two weeks of observation, 4 patients in the CG were diagnosed with pneumonia, and no one in the LG was diagnosed with pneumonia

$(\chi 2=4.248, P=0.039)$. The hospitalization days in the $L G$ was lower than that of the $C G(t=3.98, P<0.001)$

Table 5 Comparison of pneumonia and hospitalization days

\begin{tabular}{|llll|}
\hline & $\mathrm{n}$ & Pneumonia & hospitalization days \\
Group & & $\mathrm{n}(\%)$ & \\
\hline CG & 50 & $4(8)$ & $17.2 \pm 2.58$ \\
\hline LG & 51 & $0(0)$ & $15.55 \pm 1.45$ \\
\hline
\end{tabular}




\section{Discussion}

Femoral neck fractures tend to occur in elderly patients with osteoporosis. With the advent of an aging society, the number of elderly people in the world is increasing, and the number of osteoporotic patients is increasing, and the incidence of femoral neck fractures is also increasing year by year. Elderly patients with femoral neck fractures often have medical diseases. Long-term bed rest are prone to serious complications such as urinary tract infections, bedsores, pulmonary infections, and lower extremity venous thrombosis. In severe cases, they can be life-threatening. Therefore, early surgical treatment should be performed if there is no contraindication to surgery. With the widespread development of total hip arthroplasty, its surgical indications have been gradually relaxed, and more and more elderly patients undergo artificial total hip replacement. This operation can restore the function of the affected limb, improve the quality of life of the patient, avoid complications such as fracture nonunion, femoral head necrosis, and get out of bed early to reduce the complications of bed rest ${ }^{[9]}$. Elderly patients with femoral neck fractures have the following pathophysiological characteristics before surgery: lung function degradation, different degrees of ventilation or ventilation dysfunction, respiratory mucosa atrophy, cilia dyskinesia, reduced airway clearance; increased goblet cells in the airway mucosa, The secretions are many and sticky; most of them are combined with various medical diseases, which require medical treatment before surgery. The bed time is relatively long, which may cause slow recovery of respiratory function during perioperative period, decrease of respiratory muscle strength, and significant increase of lung infection. Therefore, the prevention and treatment of perioperative lung infections in elderly patients with fractures is particularly important, and one of the methods to improve respiratory function is to conduct preoperative and postoperative respiratory function training.

The application of pulmonary function breathing training device can effectively increase the FEV1, FVC and $\mathrm{PaO} 2$ of elderly patients during perioperative period, and increase the effective alveolar ventilation. The principle is that the lung function trainer can improve the uneven distribution of inhaled gas and hypoxia through training. By actively and slowly inhaling and exhaling air, reducing the breathing frequency, increasing the internal pressure of the trachea, preventing premature pressure of the bronchi and small bronchi, effectively eliminating residual lung gas, improving the ventilation / blood flow imbalance, increasing tidal volume and increasing effective ventilation The amount of functional residual gas reduces the dilution of fresh air inhaled and increases the alveolar carbon dioxide partial pressure, thereby improving gas exchange and improving the patient's ventilation function; full expansion of the thoracic cavity and increased negative pressure in the pleural cavity are conducive to lung expansion and atrophy In order to further prevent atelectasis, atelectasis or atelectasis can effectively prevent atelectasis, maximize lung function, and increase alveolar oxygen uptake capacity, thereby reducing the occurrence of hypoxemia and effectively preventing atelectasis and Pulmonary infections ${ }^{[10-11]}$.

Mills et al. ${ }^{[12]}$ conducted an 8-week inspiratory muscle training for 65-75-year-old patients and found that the strength of the inspiratory muscle was significantly improved, and pointed out that inspiratory muscle 
training in the elderly with weak and poor respiratory function groups, inspiratory The improvement of muscle function is more obvious. Moreover, inspiratory muscle training can reduce the concentration of IL-1 and IL- 6 by enhancing the function of respiratory muscles, thereby reducing the systemic inflammatory response ${ }^{[13]}$. Other scholars have also confirmed that inspiratory muscle training reduces lung infections while improving lung function, esophageal cancer, and respiratory dysfunction after abdominal surgery ${ }^{[14-16]}$. In this study, patients in the LG received routine rehabilitation training while adding pulmonary respiratory muscle function training, and it was found that respiratory function was significantly improved, and the incidence of pulmonary infection in the perioperative period was reduced. Compared with the $C G$, the difference was statistically significant $(P<0.05)$ (Table 5). The author believes that the reason may be that respiratory muscle training enhances the endurance of diaphragm and intercostal muscles, increases the alveolar oxygen carrying capacity and effective ventilation, improves respiratory function, and prevents and reduces the occurrence of lung infections.

On the basis of routine lung care, combined with respiratory muscle training can maximize the active contraction of respiratory muscles, re-mobilize the airway cilia clearance function, increase airway clearance and defense capabilities, and enhance airway mucosal function and secretion Reducing and eliminating functional enhancement is an effective way to prevent postoperative complications. Celli et al. ${ }^{[17]}$ found that compared with the control group, the comprehensive training group had significantly lower hospital stay for postoperative pulmonary complications. The results of the study in the CG and the LG showed that FEV1, FVC and SPO2 in the LG were higher than those in the $C G$ at 3 and 7 days after operation $(P<0.05)$,the difference was statistically significant. It shows that in the perioperative period, the use of lung function training equipment can effectively improve lung function and avoid pulmonary complications.

In summary, the combined use of lung function breathing exercisers on the basis of perioperative routine care for elderly patients not only quantifies the recovery of patients 'lung function, but also increases the patient's initiative and enthusiasm for lung function training, which leads to patients' Good psychological motivation, enhance patients' self-confidence and fun in respiratory function exercise, further improve lung function, shorten the waiting time for surgery and reduce postoperative respiratory complications, and effectively save medical resources.

\section{Limitations Of This Study}

The study time is relatively short, and the control time needs to be further extended to observe the treatment effect.

\section{Conclusion}

The use of lung function training devices on the basis of perioperative routine care for elderly patients with fractures can improve the lung function of patients in a short period of time, improve the patient's surgical tolerance, reduce the complications of respiratory infections, and increase the success rate of 
surgery. Shorten the length of hospitalization, reduce the financial burden of patients, and make it return to social life more quickly.

\section{Abbreviations}

FEV1: Forced expiratory volume in the first second; FVC: Forced vital capacity; MVV: Maximum minute ventilation; RV: Residual volume; TIC: Maximum minute ventilation; PaO2: Oxygen partial pressure; SPO2: Blood oxygen saturation;CG: Conventional group;LG:Lung function trainer group.

\section{Declarations}

\section{Acknowledgement}

The author would like to thank all participants for their cooperation, thank all participating experts for their help and suggestions, and thank the nurses who have directed and supervised the training program.

\section{Author's contribution}

Zhang Yan designed the protocol and wrote the manuscript. Wang Hong supervised the research. Fan Jingmei and Fan Lin collected and analyzed relevant data, and Wang Min edited the language. All authors read and approved the final manuscript.

\section{Funds}

No.

\section{Availability of data and materials}

The dataset supporting the conclusions of this article is included within the article

\section{Ethical approval and consent to participate}

This study was approved by the Ethics Committee of Zhongshan Hospital Affiliated to Dalian University, and all participants signed written informed consent.

\section{Consent for publication}

Not applicable

\section{Competing interests}

The authors declare that they have no competing interestsAuthor details.

\section{Author details}


Department of Orthopedics, Zhongshan Hospital, Dalian University, No. 6, Jiefang Street, Zhongshan District, Dalian, 116000

\section{References}

1. Rd ML. Hip fractures: a worldwide problem today and tomorrow.[J]. Bone. 1993;14(1):1-8.

2. Cummings SR, Rubin SM, Black D. The future of hip fractures in the United States. Numbers, costs, and potential effects of postmenopausal estrogen.[J]. Clinical Orthopaedics \& Related Research, 1990, 252(252):p. 163.

3. Restrepo RD, Wettstein R, Wittnebel L, et al. Incentive spirometry: 2011.[J]. Respir Care. 2011;56(10):1600-4.

4. Agostini P, Naidu B, Cieslik H, et al. Effectiveness of incentive spirometry in patients following thoracotomy and lung resection including those at high risk for developing pulmonary complications.[J]. Thorax. 2013;68(6):580.

5. Cimen S, Ozkul V, Ketenci B, et al. Daily comparison of respiratory functions between on-pump and off-pump patients undergoing CABG[J]. Eur J Cardiothorac Surg. 2003;23(4):589-94.

6. Gigliotti F, Coli C, Bianchi R, et al. Exercise Training Improves Exertional Dyspnea in Patients With COPD a,: Evidence of the Role of Mechanical Factors[J]. Chest. 2003;123(6):1794-802.

7. Aida N, Shibuya M, Yoshino K, et al. Respiratory muscle stretch gymnastics in patients with post coronary artery bypass grafting pain: impact on respiratory muscle function, activity, mood and exercise capacity[J]. Journal of Medical Dental Sciences. 2002;49(4):157.

8. Jr Heinzelman HP, Mercer M. M A. Pneumonia in the surgical patient.[J]. Curr Probl Surg. 1951;34(2):117-200.

9. Gjertsen JE, Vinje T, Engesaeter LB, et al. Internal screw fixation compared with bipolar hemiarthroplasty for treatment of displaced femoral neck fractures in elderly patients[J]. Journal of Bone \& Joint Surgery-american Volume, 2010, 92(3):pp. 619-28.

10. Evensen AE. Management of COPD exacerbations.[J]. Am Fam Physician. 2010;81(5):607-13.

11. Branson RD. The scientific basis for postoperative respiratory care[J]. Respir Care. 2013;58(11):1974-84.

12. Mills DE, Johnson MA, Mcphilimey MJ, et al. Influence of oxidative stress, diaphragm fatigue, and inspiratory muscle training on the plasma cytokine response to maximum sustainable voluntary ventilation.[J]. J Appl Physiol. 2014;116(8):970.

13. Mills DE, Johnson MA, Mcphilimey MJ, et al. The effects of inspiratory muscle training on plasma interleukin- 6 concentration during cycling exercise and a volitional mimic of the exercise hyperpnea. [J]. J Appl Physiol. 2013;115(8):1163-72.

14. Tout $R$, Tayara $L$, Halimi $M$. The effects of respiratory muscle training on improvement of the internal and external thoraco-pulmonary respiratory mechanism in COPD patients[J]. Annals of Physical Rehabilitation Medicine. 2013;56(3):193-211. 
15. Katsura M, Kuriyama A, Takeshima T, et al. Preoperative inspiratory muscle training for postoperative pulmonary complications in adults undergoing cardiac and major abdominal surgery[J]. Cochrane Database of Systematic Reviews. 2015;10(10):CD010356.

16. Mans CM, Reeve JC, Gasparini CA, et al. Postoperative outcomes following preoperative inspiratory muscle training in patients undergoing open cardiothoracic or upper abdominal surgery: protocol for a systematic review[J]. Systematic Reviews. 2012;1(1):63.

17. Celli BR, Rodriguez KS, Snider GL. A controlled trial of intermittent positive pressure breathing, incentive spirometry, and deep breathing exercises in preventing pulmonary complications after abdominal surgery.[J]. Am Rev Respir Dis. 1984;130(1):12-5. 